Turk. J. Math. Comput. Sci.

13(2)(2021) 379-387

(C) MatDer

DOI : $10.47000 /$ tjmcs. 969243

\title{
Some Characterizations of Spherical Indicatrix Curves Generated by Flc Frame
}

\author{
Süleyman Şenyurt ${ }^{1}$ (D), Kebire Hilal Ayvaci ${ }^{1, *}$ (D), Davut Canli ${ }^{1}$ (D) \\ ${ }^{1}$ Department of Mathematics, Ordu University, Cumhuriyet Yerleşkesi, Ordu, Turkey.
}

Received: 09-07-2021 • Accepted: 13-08-2021

\begin{abstract}
AвSTRACT. In this study, the spherical indicatrices of Flc frame vectors were defined on unit sphere. The arc length parameters and the Frenet vectors of these indicatrix curves were calculated, as well. Last, we have provided the geodesic curvatures according to both Euclidean space $E^{3}$ and unit sphere $S^{2}$.
\end{abstract}

2010 AMS Classification: 53A04, 53A05

Keywords: Polynomial curves, Flc frame, spherical indicatrix, geodesic curvature.

\section{INTRODUCTION}

In classical differential geometry, the curves are characterized by a moving frame of which the most referred one is the Frenet frame. However, to settle a frame on a given curve like Frenet, it is required to make a choice of at least $C^{3}$ class non-degenerate curve. In 1975, L.Bishop defined another frame known to be alternative parallel frame that can be settled on the points at which even the curvatures vanish [1]. As Bishop pointed out in his study that "there is more than one way to frame a curve", recently Dede has introduced a new moving frame relatively having some advantages but under the constraint that the curve to be framed should be polynomial. They named it as the Flc (Frenet-like curve) frame. Calculations to be made for Flc frame is much easier compared to Frenet frame and it has an analytic form which is not the case for Bishop frame. In addition to these, Flc frame is less singular than Frenet frame and has much less undesirable rotations around the tangent of the curve [3]. Spherical indicatrices, on the other hand, are special curves drawn by the unit vectors centered at a unit sphere. In 3 dimensional Euclidean space, the characteristics of such curves like arc-lengths and geodesic curvatures are given in [6]. These are examined for Minkowski space in [2]. The spherical indicatrix curves according to Bishop frame and Dual Bishop frame were investigated in [12] and [5], respectively. The spherical indicatrices were charecterized on slant helix as a special curve in [7]. There are also studies spherical indicatrices curves of some special curves in [10,11].

In this study, we first defined the spherical indicatrix curves of each vector components of Flc frame on unit sphere. Then for each spherical indicatrices, we calculated the Frenet frame and defined the arc lengths and the geodesic curvatures for both $E^{3}$ and $S^{2}$.

*Corresponding Author

Email addresses: senyurtsuleyman52@gmail.com (S. Şenyurt), kebirehilalayvaci@odu.edu.tr (K.H. Ayvacı), davutcanli@odu.edu.tr (D. Canlı) 


\section{Preliminaries}

In this section, we remind some basic concepts that will be used through out the paper. Let $\alpha=\alpha(s)$ be a regular space curve and non-degenerate condition $\alpha^{\prime}(s) \wedge \alpha^{\prime \prime}(s) \neq 0$. Then, three orthogonal vector fields which called Frenet frame are defined as [9]

$$
T(s)=\frac{\alpha^{\prime}(s)}{\left\|\alpha^{\prime}(s)\right\|}, \quad B(s)=\frac{\alpha^{\prime}(s) \wedge \alpha^{\prime \prime}(s)}{\left\|\alpha^{\prime}(s) \wedge \alpha^{\prime \prime}(s)\right\|}, \quad N(s)=B(s) \wedge T(s),
$$

where $T$ is the tangent, $N$ is the principal normal and $B$ is the binormal vector field. The Frenet formulas are given by

$$
T^{\prime}=\kappa \nu N, \quad N^{\prime}=-\kappa v T+\tau \nu B, B^{\prime}=-\tau v N,\left\|\alpha^{\prime}\right\|=v,
$$

where the curvature $\kappa$ and torsion $\tau$ of the curve are

$$
\kappa=\frac{\left\|\alpha^{\prime}(s) \wedge \alpha^{\prime \prime}(s)\right\|}{\left\|\alpha^{\prime}(s)\right\|^{3}}, \quad \tau=\frac{\left\langle\alpha^{\prime}(s) \wedge \alpha^{\prime \prime}(s), \alpha^{\prime \prime \prime}(s)\right\rangle}{\left\|\alpha^{\prime}(s) \wedge \alpha^{\prime \prime}(s)\right\|^{2}} .
$$

We define the $n^{\text {th }}$ degree polynomial with parameter $t$ as

$$
P(t)=a_{n} t^{n}+a_{n-1} t^{n-1}+\ldots+a_{1} t^{1}+a_{0}, \quad a_{n} \neq 0,
$$

where $n \in N_{0}, a_{i} \in \mathbb{R},(0 \leq i \leq n)[8]$.

Now let us define a curve such that, $\alpha:[a, b] \rightarrow E^{n}, \alpha(t)=\left(\alpha_{1}(t), \alpha_{2}(t), \ldots, \alpha_{n}(t)\right)$. If each $\alpha_{i}$ s are polynomial for $1 \leq i \leq n$, then $a_{t} \in \mathbb{R}[t]$ is defined to be an n-dimensional polynomial curve [4]. The degree of such a polynomial curve as $\alpha(t)$ is given by

$$
\operatorname{deg} \alpha(t)=\max \left\{\operatorname{deg}\left(\alpha_{1}(t)\right), \operatorname{deg}\left(\alpha_{2}(t)\right), \ldots, \operatorname{deg}\left(\alpha_{n}(t)\right)\right\} \quad[8] .
$$

Regarding to these, let us define $\alpha=\alpha(s)$ as a polynomial space curve. The definition of the Flc frame for $\alpha$ introduced by Dede in [3] is as following

$$
T(s)=\frac{\alpha^{\prime}(s)}{\left\|\alpha^{\prime}(s)\right\|}, \quad D_{1}(s)=\frac{\alpha^{\prime}(s) \wedge \alpha^{(n)}(s)}{\left\|\alpha^{\prime}(s) \wedge \alpha^{(n)}(s)\right\|}, \quad D_{2}(s)=D_{1}(s) \wedge T(s),
$$

where the prime' indicates the differentiation with respect to $s$ and ${ }^{(n)}$ stands for the $n^{\text {th }}$ derivative. The new vectors $D_{1}$ and $D_{2}$ are called as binormal-like vector and normal-like vector, respectively. The curvatures of the Flc-frame $d_{1}, d_{2}$ and $d_{3}$ are given by

$$
d_{1}=\frac{\left\langle T^{\prime}, D_{2}\right\rangle}{v}, d_{2}=\frac{\left\langle T^{\prime}, D_{1}\right\rangle}{v}, d_{3}=\frac{\left\langle D_{2}{ }^{\prime}, D_{1}\right\rangle}{v},
$$

where $\left\|\alpha^{\prime}\right\|=v$. The local rate of change of the Flc-frame called as the Frenet-like formulas can be expressed in the following form

$$
\left[\begin{array}{c}
T^{\prime} \\
D_{2}^{\prime} \\
D_{1}^{\prime}
\end{array}\right]=v\left[\begin{array}{ccc}
0 & d_{1} & d_{2} \\
-d_{1} & 0 & d_{3} \\
-d_{2} & -d_{3} & 0
\end{array}\right]\left[\begin{array}{c}
T \\
D_{2} \\
D_{1}
\end{array}\right] .
$$

The Darboux vector $D_{W}$ of the Flc-Frame can be obtained as in the following form

$$
D_{W}=v\left(d_{3} T-d_{2} D_{2}+d_{1} D_{1}\right)
$$

Thus, the instantaneous angular speed of the Flc-frame is calculated as follows

$$
\left\|D_{W}\right\|=v \sqrt{d_{1}^{2}+d_{2}^{2}+d_{3}^{2}} .
$$

The instantaneous rate of change for each of these vectors $T, D_{2}$ and $D_{1}$ has two components, namely from (2.5), we write

- $D_{W} \wedge T=d_{2} D_{1}+d_{1} D_{2}$, thus $T$ changes instantaneous rate $d_{2}$ and $d_{1}$ in the direction of $D_{2}$ and $D_{1}$, respectively

- $D_{W} \wedge D_{2}=d_{3} D_{1}-d_{1} T$, thus $D_{2}$ changes instantaneous rate $d_{3}$ and $d_{1}$ in the direction of $D_{2}$ and $T$, respectively.

- $D_{W} \wedge D_{1}=-d_{3} D_{2}-d_{2} T$, thus $D_{1}$ changes instantaneous rate $d_{3}$ and $d_{2}$ in the direction of $D_{2}$ and $T$, respectively. 
For a surface, $M$ in $E^{3}$, let us denote the shape operator with $S$, the unit normal vector field with $\xi$ and the Riemann connection with $D$, then for $\forall X, Y \in \chi(M)$, the Gauss equation is defined by

$$
\bar{D}_{X} Y=D_{X} Y+\langle S(X), Y\rangle \xi .
$$

Here, $\bar{D}$ is derivative operator in Gauss sense.

The geodesic curvatures of $\alpha$ according to $E^{3}$ and $S^{2}$ is given by

$$
\begin{aligned}
& k_{g}=\left\|D_{T} T\right\|, \\
& \mu_{g}=\left\|\bar{D}_{T} T\right\|,
\end{aligned}
$$

respectively [6].

\section{Some Characterizations of Spherical Indicatrix Curves Generated by Flc Frame}

In this section, we define the spherical indicatrix curves of Flc frame on the unit sphere. We also provide the Frenet elements of each indicatrices and by using Gauss equation we calculate geodesic curvatures according to both $E^{3}$ and $S^{2}$.

Definition 3.1. Let $\alpha$ be a polynomial curve in $E^{3}$ and $\left\{T, D_{2}, D_{1}\right\}$ denote its Flc frame. The curve traced out by the vector $T$ centered at unit sphere is called $T$ - or tangent indicatrix curve according to Flc frame and is defined as

$$
\alpha_{T}(s)=T(s) .
$$

Theorem 3.2. The Frenet vectors $T_{T}, N_{T}, B_{T}$ of $T$-indicatrix curve according to Flc frame are given as follows:

$$
\begin{aligned}
& T_{T}=\frac{d_{1}}{\sqrt{d_{1}^{2}+d_{2}^{2}}} D_{2}+\frac{d_{2}}{\sqrt{d_{1}^{2}+d_{2}^{2}}} D_{1}, \\
& N_{T}=\frac{x_{2} d_{2}-x_{3} d_{1}}{\sqrt{d_{1}^{2}+d_{2}^{2}}} T-\frac{x_{1} d_{2}}{\sqrt{d_{1}^{2}+d_{2}^{2}}} D_{2}+\frac{x_{1} d_{1}}{\sqrt{d_{1}^{2}+d_{2}^{2}}} D_{1} \\
& B_{T}=\frac{\left(v^{3} d_{3}\left(d_{1}^{2}+d_{2}^{2}\right)+v d_{1}\left(v d_{2}\right)^{\prime}-v d_{2}\left(v d_{1}\right)^{\prime}\right) T-\left(v^{3} d_{2}\left(d_{1}^{2}+d_{2}^{2}\right)\right) D_{2}+\left(v^{3} d_{1}\left(d_{1}^{2}+d_{2}^{2}\right)\right) D_{1}}{\sqrt{\left(v^{3} d_{3}\left(d_{1}^{2}+d_{2}^{2}\right)+v d_{1}\left(v d_{2}\right)^{\prime}-v d_{2}\left(v d_{1}\right)^{\prime}\right)^{2}+v^{6}\left(d_{1}^{2}+d_{2}^{2}\right)^{3}}},
\end{aligned}
$$

where $x_{1}, x_{2}, x_{3}$ are coefficients of the following form

$$
\begin{aligned}
& x_{1}=\frac{v^{3} d_{3}\left(d_{1}^{2}+d_{2}^{2}\right)+v d_{1}\left(v d_{2}\right)^{\prime}-v d_{2}\left(v d_{1}\right)^{\prime}}{\sqrt{\left(v^{3} d_{3}\left(d_{1}^{2}+d_{2}^{2}\right)+v d_{1}\left(v d_{2}\right)^{\prime}-v d_{2}\left(v d_{1}\right)^{\prime}\right)^{2}+v^{6}\left(d_{1}^{2}+d_{2}^{2}\right)^{3}}}, \\
& x_{2}=-\frac{v^{3} d_{2}\left(d_{1}^{2}+d_{2}^{2}\right)}{\sqrt{\left(v^{3} d_{3}\left(d_{1}^{2}+d_{2}^{2}\right)+v d_{1}\left(v d_{2}\right)^{\prime}-v d_{2}\left(v d_{1}\right)^{\prime}\right)^{2}+v^{6}\left(d_{1}^{2}+d_{2}^{2}\right)^{3}}},, \\
& x_{3}=\frac{v^{3} d_{1}\left(d_{1}^{2}+d_{2}^{2}\right)}{\sqrt{\left(v^{3} d_{3}\left(d_{1}^{2}+d_{2}^{2}\right)+v d_{1}\left(v d_{2}\right)^{\prime}-v d_{2}\left(v d_{1}\right)^{\prime}\right)^{2}+v^{6}\left(d_{1}^{2}+d_{2}^{2}\right)^{3}}} .
\end{aligned}
$$

Proof. By referring the relation (2.4), the first and second derivative of $\alpha_{T}(s)=T(s)$ can be easily given as

$$
\begin{aligned}
\alpha_{T}{ }^{\prime}(s) & =v\left(d_{1} D_{2}+d_{2} D_{1}\right), \\
\alpha_{T}{ }^{\prime \prime}(s) & =-v^{2}\left(d_{1}^{2}+d_{2}^{2}\right) T+\left(\left(v d_{1}\right)^{\prime}-v^{2} d_{2} d_{3}\right) D_{2}+\left(\left(v d_{2}\right)^{\prime}+v^{2} d_{1} d_{3}\right) D_{1} .
\end{aligned}
$$


Now, recalling the definitions given in (2.1) and (2.2), we calculate the required components as

$$
\begin{aligned}
& \left\|\alpha_{T}{ }^{\prime}(s)\right\|=v \sqrt{d_{1}^{2}+d_{2}^{2}} \\
& \alpha_{T}^{\prime}(s) \wedge \alpha_{T}^{\prime \prime}(s)=\left(v^{3} d_{3}\left(d_{1}^{2}+d_{2}^{2}\right)+v d_{1}\left(v d_{2}\right)^{\prime}-v d_{2}\left(v d_{1}\right)^{\prime}\right) T-\left(v^{3} d_{2}\left(d_{1}^{2}+d_{2}^{2}\right)\right) D_{2}+\left(v^{3} d_{1}\left(d_{1}^{2}+d_{2}^{2}\right)\right) D_{1} \\
& \left\|\alpha_{T}^{\prime}(s) \wedge \alpha_{T}^{\prime \prime}(s)\right\|=\sqrt{\left(v^{3} d_{3}\left(d_{1}^{2}+d_{2}^{2}\right)+v d_{1}\left(v d_{2}\right)^{\prime}-v d_{2}\left(v d_{1}\right)^{\prime}\right)^{2}+v^{6}\left(d_{1}^{2}+d_{2}^{2}\right)^{3}}
\end{aligned}
$$

Substituting these relations in place we complete the proof.

Theorem 3.3. The geodesic curvatures of $T$ - indicatrix curve according to $E^{3}$ and $S^{2}$ are given as following:

$$
\begin{aligned}
& k_{g}^{T}=\frac{\sqrt{\left(d_{1}{ }^{2}+d_{2}{ }^{2}\right)\left(d_{1} d_{2}{ }^{\prime}-d_{2} d_{1}{ }^{\prime}\right)^{2}+2 d_{3}\left(d_{1} d_{2}{ }^{\prime}-d_{2} d_{1}{ }^{\prime}\right)\left(d_{1}{ }^{2}+d_{2}{ }^{2}\right)^{2}+d_{3}{ }^{2}\left(d_{1}{ }^{2}+d_{2}{ }^{2}\right)^{3}+\left(d_{1}{ }^{2}+d_{2}{ }^{2}\right)^{4}}}{\left(d_{1}{ }^{2}+d_{2}{ }^{2}\right)^{2}}, \\
& \mu_{g}^{T}=\frac{\sqrt{\left(d_{1}{ }^{2}+d_{2}{ }^{2}\right)\left(d_{1} d_{2}{ }^{\prime}-d_{2} d_{1}{ }^{\prime}\right)^{2}+2 d_{3}\left(d_{1} d_{2}{ }^{\prime}-d_{2} d_{1}{ }^{\prime}\right)\left(d_{1}{ }^{2}+d_{2}{ }^{2}\right)^{2}+d_{3}{ }^{2}\left(d_{1}{ }^{2}+d_{2}{ }^{2}\right)^{3}}}{\left(d_{1}{ }^{2}+d_{2}{ }^{2}\right)^{2}},
\end{aligned}
$$

respectively.

Proof. From (3.1), if we take the derivative of $T_{T}$ and consider the relation (2.4), then we get

$$
D_{T_{T}} T_{T}=\frac{-\left(d_{2}\left(d_{1} d_{2}{ }^{\prime}-d_{2} d_{1}{ }^{\prime}\right)+d_{2} d_{3}\left(d_{1}^{2}+d_{2}{ }^{2}\right)\right) D_{2}+\left(d_{1}\left(d_{1} d_{2}{ }^{\prime}-d_{2} d_{1}{ }^{\prime}\right)+d_{1} d_{3}\left(d_{1}{ }^{2}+d_{2}{ }^{2}\right)\right) D_{1}}{\left(d_{1}{ }^{2}+d_{2}{ }^{2}\right)^{2}}-T .
$$

By using the relation given in (2.7), $k_{g}^{T}$ can be easily obtained. On the other hand, when considered the Gauss equation given in (2.6), we get the following relations

$$
\begin{aligned}
\bar{D}_{T_{T}} T_{T} & =D_{T_{T}} T_{T}+\left\langle S\left(T_{T}\right), T_{T}\right\rangle T \\
& =\frac{-\left(d_{2}\left(d_{1} d_{2}{ }^{\prime}-d_{2} d_{1}{ }^{\prime}\right)+d_{2} d_{3}\left(d_{1}{ }^{2}+d_{2}{ }^{2}\right)\right) D_{2}+\left(d_{1}\left(d_{1} d_{2}{ }^{\prime}-d_{2} d_{1}{ }^{\prime}\right)+d_{1} d_{3}\left(d_{1}{ }^{2}+d_{2}{ }^{2}\right)\right) D_{1}}{\left(d_{1}{ }^{2}+d_{2}{ }^{2}\right)^{2}}
\end{aligned}
$$

to be used to calculate $\mu_{g}^{T}$ by the definition given in (2.8), which completes the proof.

Remark 3.4. Since, Frenet and Flc frame have a common tangent vector field which means the images of tangent indicatrix for both are the same on a unit sphere, one would argue the necessity of calculations. However, we are concerned to the Flc frame, and express those characterizations of T-indicatrix according to the Flc invariants.

Definition 3.5. Let $\alpha$ be a polynomial curve in $E^{3}$ and $\left\{T, D_{2}, D_{1}\right\}$ denote the Flc frame. The curve traced out by the vector $D_{2}$ centered at unit sphere is called $D_{2}-$ or normal like indicatrix curve and is defined as

$$
\alpha_{D_{2}}(s)=D_{2}(s)
$$

Theorem 3.6. The Frenet vectors $T_{D_{2}}, N_{D_{2}}, B_{D_{2}}$ of $D_{2}$ - indicatrix curve according to Flc frame are given as follows: 


$$
\begin{aligned}
& T_{D_{2}}=\frac{-d_{1}}{\sqrt{d_{1}^{2}+d_{3}^{2}}} T+\frac{d_{3}}{\sqrt{d_{1}^{2}+d_{3}^{2}}} D_{1}, \\
& N_{D_{2}}=\frac{u_{2} d_{3}}{\sqrt{d_{1}^{2}+d_{3}^{2}}} T-\frac{u_{1} d_{3}+u_{3} d_{1}}{\sqrt{d_{1}^{2}+d_{3}^{2}}} D_{2}+\frac{u_{2} d_{1}}{\sqrt{d_{1}^{2}+d_{3}^{2}}} D_{1}, \\
& B_{D_{2}}=\frac{\left(v^{3} d_{3}\left(d_{1}^{2}+d_{3}^{2}\right)\right) T+\left(-v d_{3}\left(\left(v d_{1}\right)^{\prime}+d_{2} d_{3} v^{2}\right)+v d_{1}\left(\left(v d_{3}\right)^{\prime}-d_{1} d_{2} v^{2}\right)\right) D_{2}+\left(v^{3} d_{1}\left(d_{1}^{2}+d_{3}^{2}\right)\right) D_{1}}{\sqrt{\left(-v d_{3}\left(\left(v d_{1}\right)^{\prime}+d_{2} d_{3} v^{2}\right)+v d_{1}\left(\left(v d_{3}\right)^{\prime}-d_{1} d_{2} v^{2}\right)\right)^{2}+v^{6}\left(d_{1}^{2}+d_{3}^{2}\right)^{3}}}
\end{aligned}
$$

where the coefficients $u_{1}, u_{2}$ and $u_{3}$ are of the following forms:

$$
\begin{aligned}
& u_{1}=\frac{v^{3} d_{3}\left(d_{1}^{2}+d_{3}^{2}\right)}{\sqrt{\left(-v d_{3}\left(\left(v d_{1}\right)^{\prime}+d_{2} d_{3} v^{2}\right)+v d_{1}\left(\left(v d_{3}\right)^{\prime}-d_{1} d_{2} v^{2}\right)\right)^{2}+v^{6}\left(d_{1}^{2}+d_{3}^{2}\right)^{3}}}, \\
& u_{2}=\frac{-v d_{3}\left(\left(v d_{1}\right)^{\prime}+d_{2} d_{3} v^{2}\right)+v d_{1}\left(\left(v d_{3}\right)^{\prime}-d_{1} d_{2} v^{2}\right)}{\sqrt{\left(-v d_{3}\left(\left(v d_{1}\right)^{\prime}+d_{2} d_{3} v^{2}\right)+v d_{1}\left(\left(v d_{3}\right)^{\prime}-d_{1} d_{2} v^{2}\right)\right)^{2}+v^{6}\left(d_{1}^{2}+d_{3}^{2}\right)^{3}}}, \\
& u_{3}=\frac{v^{3} d_{1}\left(d_{1}^{2}+d_{3}^{2}\right)}{\sqrt{\left(-v d_{3}\left(\left(v d_{1}\right)^{\prime}+d_{2} d_{3} v^{2}\right)+v d_{1}\left(\left(v d_{3}\right)^{\prime}-d_{1} d_{2} v^{2}\right)\right)^{2}+v^{6}\left(d_{1}^{2}+d_{3}^{2}\right)^{3}}},
\end{aligned}
$$

respectively.

Proof. By taking the first and second derivative of the expression $\alpha_{D_{2}}(s)=D_{2}(s)$ and using (2.4) we get

$$
\begin{aligned}
& \alpha_{D_{2}}{ }^{\prime}(s)=v\left(-d_{1} T+d_{3} D_{1}\right), \\
& \alpha_{D_{2}}{ }^{\prime \prime}(s)=-\left(\left(v d_{1}\right)^{\prime}+d_{2} d_{3} v^{2}\right) T-v^{2}\left(d_{1}^{2}+d_{3}^{2}\right) D_{2}+\left(\left(v d_{3}\right)^{\prime}-d_{1} d_{2} v^{2}\right) D_{1} .
\end{aligned}
$$

Similarly, by calculating the necessary relations as following

$$
\begin{aligned}
& \left\|\alpha_{D_{2}}{ }^{\prime}(s)\right\|=v \sqrt{d_{1}{ }^{2}+d_{3}{ }^{2}}, \\
& \alpha_{D_{2}}{ }^{\prime}(s) \wedge \alpha_{D_{2}}{ }^{\prime \prime}(s)=\left(v^{3} d_{3}\left(d_{1}^{2}+d_{3}^{2}\right)\right) T+\left(-v d_{3}\left(\left(v d_{1}\right)^{\prime}+d_{2} d_{3} v^{2}\right)+v d_{1}\left(\left(v d_{3}\right)^{\prime}-d_{1} d_{2} v^{2}\right)\right) D_{2}+\left(v^{3} d_{1}\left(d_{1}^{2}+d_{3}^{2}\right)\right) D_{1}, \\
& \left\|\alpha_{D_{2}}{ }^{\prime}(s) \wedge \alpha_{D_{2}}{ }^{\prime \prime}(s)\right\|=\sqrt{\left(-v d_{3}\left(\left(v d_{1}\right)^{\prime}+d_{2} d_{3} v^{2}\right)+v d_{1}\left(\left(v d_{3}\right)^{\prime}-d_{1} d_{2} v^{2}\right)\right)^{2}+v^{6}\left(d_{1}^{2}+d_{3}^{2}\right)^{3}}
\end{aligned}
$$

to substitute in (2.1), we complete the proof.

Theorem 3.7. The geodesic curvatures of $D_{2}$ - indicatrix curve according to $E^{3}$ and $S^{2}$ are given as following:

$$
\begin{aligned}
k_{g}^{D_{2}} & =\frac{\sqrt{\left(d_{1}{ }^{2}+d_{3}{ }^{2}\right)\left(d_{1} d_{3}{ }^{\prime}-d_{3} d_{1}{ }^{\prime}\right)^{2}-2 d_{2}\left(d_{1} d_{3}{ }^{\prime}-d_{3} d_{1}{ }^{\prime}\right)\left(d_{1}{ }^{2}+d_{3}{ }^{2}\right)^{2}+d_{2}{ }^{2}\left(d_{1}{ }^{2}+d_{3}{ }^{2}\right)^{3}+\left(d_{1}{ }^{2}+d_{3}{ }^{2}\right)^{4}}}{\left(d_{1}{ }^{2}+d_{3}{ }^{2}\right)^{2}}, \\
\mu_{g}^{D_{2}} & =\frac{\sqrt{\left(d_{1}{ }^{2}+d_{3}{ }^{2}\right)\left(d_{1} d_{3}{ }^{\prime}-d_{3} d_{1}{ }^{\prime}\right)^{2}-2 d_{2}\left(d_{1} d_{3}{ }^{\prime}-d_{3} d_{1}{ }^{\prime}\right)\left(d_{1}{ }^{2}+d_{3}{ }^{2}\right)^{2}+d_{2}{ }^{2}\left(d_{1}{ }^{2}+d_{3}{ }^{2}\right)^{3}}}{\left(d_{1}{ }^{2}+d_{3}{ }^{2}\right)^{2}},
\end{aligned}
$$


respectively.

Proof. By taking the derivative of $T_{D_{2}}$ given in (3.2), and considering again (2.4), we have

$$
D_{T_{D_{2}}} T_{D_{2}}=\frac{\left(d_{3}\left(d_{1} d_{3}{ }^{\prime}-d_{3} d_{1}{ }^{\prime}\right)-d_{2} d_{3}\left(d_{1}{ }^{2}+d_{3}{ }^{2}\right)\right) T+\left(d_{1}\left(d_{1} d_{3}{ }^{\prime}-d_{3} d_{1}{ }^{\prime}\right)-d_{1} d_{2}\left(d_{1}{ }^{2}+d_{3}{ }^{2}\right)\right) D_{1}}{\left(d_{1}{ }^{2}+d_{3}{ }^{2}\right)^{2}}-D_{2} .
$$

From the definition, (2.7), the geodesic curvature according to $E^{3}, k_{g}^{D_{2}}$ can be easily calculated. By the Gauss relation (2.6), we write

$$
\begin{aligned}
\bar{D}_{T_{D_{2}}} T_{D_{2}} & =D_{T_{D_{2}}} T_{D_{2}}+\left\langle S\left(T_{D_{2}}\right), T_{D_{2}}\right\rangle D_{2} \\
& =\frac{\left(d_{3}\left(d_{1} d_{3}{ }^{\prime}-d_{3} d_{1}{ }^{\prime}\right)-d_{2} d_{3}\left(d_{1}{ }^{2}+d_{3}{ }^{2}\right)\right) T+\left(d_{1}\left(d_{1} d_{3}{ }^{\prime}-d_{3} d_{1}{ }^{\prime}\right)-d_{1} d_{2}\left(d_{1}{ }^{2}+d_{3}{ }^{2}\right)\right) D_{1}}{\left(d_{1}{ }^{2}+d_{3}{ }^{2}\right)^{2}} .
\end{aligned}
$$

Now, by referring the definition in (2.8) the geodesic curvature according to $S^{2}, \mu_{g}^{D_{2}}$ can be obtained, which completes the proof.

Definition 3.8. Let $\alpha$ be a polynomial curve in $E^{3}$ and $\left\{T, D_{2}, D_{1}\right\}$ denote its Flc frame. The curve traced out by the vector $D_{1}$ centered at unit sphere is called $D_{1}-$ or binormal like indicatrix curve and is defined as

$$
\alpha_{D_{1}}(s)=D_{1}(s) .
$$

Theorem 3.9. The Frenet vectors $T_{D_{1}}, N_{D_{1}}, B_{D_{1}}$ of $D_{1}$ - indicatrix curve according to Flc frame are given as follows:

$$
\begin{aligned}
& T_{D_{1}}=-\frac{d_{2}}{\sqrt{d_{2}^{2}+d_{3}^{2}}} T-\frac{d_{3}}{\sqrt{d_{2}^{2}+d_{3}^{2}}} D_{2}, \\
& N_{D_{1}}=\frac{m_{3} d_{3}}{\sqrt{d_{2}^{2}+d_{3}^{2}}} T-\frac{m_{3} d_{2}}{\sqrt{d_{2}^{2}+d_{3}^{2}}} D_{2}+\frac{m_{2} d_{2}-m_{1} d_{3}}{\sqrt{d_{2}^{2}+d_{3}^{2}}} D_{1}, \\
& B_{D_{1}}=\frac{\left(v^{3} d_{3}\left(d_{2}^{2}+d_{3}^{2}\right)\right) T-\left(v^{3} d_{2}\left(d_{2}^{2}+d_{3}^{2}\right)\right) D_{2}+\left(v d_{2}\left(\left(v d_{3}\right)^{\prime}-v d_{3}\left(v d_{2}\right)^{\prime}+v^{2} d_{1}\left(d_{2}^{2}+d_{3}^{2}\right)\right) D_{1}\right.}{\sqrt{v^{6}\left(d_{2}^{2}+d_{3}^{2}\right)^{3}+\left(v d_{2}\left(\left(v d_{3}\right)^{\prime}-v d_{3}\left(v d_{2}\right)^{\prime}+v^{2} d_{2}\left(d_{2}^{2}+d_{3}^{2}\right)\right)^{2}\right.},}
\end{aligned}
$$

where the coefficients $m_{1}, m_{2}$ and $m_{3}$ are of the following form

$$
\begin{aligned}
& m_{1}=\frac{v^{3} d_{3}\left(d_{2}^{2}+d_{3}^{2}\right)}{\sqrt{v^{6}\left(d_{2}^{2}+d_{3}^{2}\right)^{3}+\left(v d_{2}\left(\left(v d_{3}\right)^{\prime}-v d_{3}\left(v d_{2}\right)^{\prime}+v^{2} d_{2}\left(d_{2}^{2}+d_{3}^{2}\right)\right)^{2}\right.}}, \\
& m_{2}=-\frac{v^{3} d_{2}\left(d_{2}^{2}+d_{3}^{2}\right)}{\sqrt{v^{6}\left(d_{2}^{2}+d_{3}^{2}\right)^{3}+\left(v d_{2}\left(\left(v d_{3}\right)^{\prime}-v d_{3}\left(v d_{2}\right)^{\prime}+v^{2} d_{2}\left(d_{2}^{2}+d_{3}^{2}\right)\right)^{2}\right.}}, \\
& m_{3}=\frac{v d_{2}\left(\left(v d_{3}\right)^{\prime}-v d_{3}\left(v d_{2}\right)^{\prime}+v^{2} d_{1}\left(d_{2}^{2}+d_{3}^{2}\right)\right.}{\sqrt{v^{6}\left(d_{2}^{2}+d_{3}^{2}\right)^{3}+\left(v d_{2}\left(\left(v d_{3}\right)^{\prime}-v d_{3}\left(v d_{2}\right)^{\prime}+v^{2} d_{2}\left(d_{2}^{2}+d_{3}^{2}\right)\right)^{2}\right.}} .
\end{aligned}
$$

Proof. By taking the first and second derivative of the expression $\alpha_{D_{1}}(s)=D_{1}(s)$ and using (2.4) we get

$$
\begin{aligned}
& \alpha_{D_{1}}{ }^{\prime}(s)=-v\left(d_{2} T+d_{3} D_{2}\right), \\
& \alpha_{D_{1}}{ }^{\prime \prime}(s)=-\left(\left(v d_{2}\right)^{\prime}-v d_{1} d_{3}\right) T-\left(\left(v d_{3}\right)^{\prime}+v d_{1} d_{2}\right) D_{2}-v^{2}\left(d_{2}^{2}+d_{3}^{2}\right) D_{1} .
\end{aligned}
$$


By following the same steps as before, we calculate the necessary relations as

$$
\begin{gathered}
\left\|\alpha_{D_{1}}{ }^{\prime}(s)\right\|=v \sqrt{d_{2}{ }^{2}+d_{3}{ }^{2}} \\
\alpha_{D_{1}}{ }^{\prime}(s) \wedge \alpha_{D_{1}}{ }^{\prime \prime}(s)= \\
\left\|\alpha_{D_{1}}{ }^{\prime}(s) \wedge \alpha_{D_{1}}{ }^{\prime \prime} d_{3}(s)\right\|=\sqrt{\left.v^{6}\left(d_{2}^{2}+d_{3}^{2}\right)\right) T-\left(d ^ { 3 } d ^ { 2 } d _ { 2 } \left(d_{2}^{2}+\left(v d_{2}\left(\left(v d_{3}\right)^{\prime}\right) D_{2}+\left(v d_{2}\left(\left(v d_{3}\right)^{\prime}-v d_{3}\left(v d_{2}\right)^{\prime}+v^{\prime}+v^{2} d_{2}\left(d_{2}{ }^{2}+d_{2}^{2}+d_{3}^{2}\right)\right)^{2}\right.\right.\right.\right.}
\end{gathered}
$$

to substitute in (2.1) and complete the proof.

Theorem 3.10. The geodesic curvatures of $D_{1}-$ indicatrix curve according to $E^{3}$ and $S^{2}$ are given as following:

$$
\begin{aligned}
& k_{g}^{D_{1}}=\frac{\sqrt{\left(d_{2}{ }^{2}+d_{3}{ }^{2}\right)\left(d_{2} d_{3}{ }^{\prime}-d_{3} d_{2}{ }^{\prime}\right)^{2}+2 d_{1}\left(d_{2} d_{3}{ }^{\prime}-d_{3} d_{2}{ }^{\prime}\right)\left(d_{2}{ }^{2}+d_{3}{ }^{2}\right)^{2}+d_{1}{ }^{2}\left(d_{2}{ }^{2}+d_{3}{ }^{2}\right)^{3}+\left(d_{2}{ }^{2}+d_{3}{ }^{2}\right)^{4}}}{\left(d_{2}{ }^{2}+d_{3}{ }^{2}\right)^{2}}, \\
& \mu_{g}^{D_{1}}=\frac{\sqrt{\left(d_{2}^{2}+d_{3}{ }^{2}\right)\left(d_{2} d_{3}{ }^{\prime}-d_{3} d_{2}{ }^{\prime}\right)^{2}+2 d_{1}\left(d_{2} d_{3}{ }^{\prime}-d_{3} d_{2}{ }^{\prime}\right)\left(d_{2}{ }^{2}+d_{3}{ }^{2}\right)^{2}+d_{1}{ }^{2}\left(d_{2}{ }^{2}+d_{3}{ }^{2}\right)^{3}}}{\left(d_{2}^{2}+d_{3}{ }^{2}\right)^{2}},
\end{aligned}
$$

respectively.

Proof. By taking the derivative of $T_{D_{1}}$ given in (3.3), and recalling once more (2.4), we have

$$
D_{T_{D_{1}}} T_{D_{1}}=\frac{\left(d_{3}\left(d_{2} d_{3}{ }^{\prime}-d_{3} d_{2}{ }^{\prime}\right)+d_{1} d_{3}\left(d_{2}^{2}+d_{3}{ }^{2}\right)\right) T+\left(d_{2}\left(d_{3} d_{2}{ }^{\prime}-d_{2} d_{3}{ }^{\prime}\right)-d_{1} d_{2}\left(d_{2}^{2}+d_{3}{ }^{2}\right)\right) D_{2}}{\left(d_{2}{ }^{2}+d_{3}{ }^{2}\right)^{2}}-D_{1} .
$$

From the definition in (2.7), $k_{g}^{D_{1}}$ is simply the norm of latter relation. By recalling the Gauss equation (2.6) again, we have

$$
\begin{aligned}
\bar{D}_{T_{D_{1}}} T_{D_{1}} & =D_{T_{D_{1}}} T_{D_{1}}+\left\langle S\left(T_{D_{1}}\right), T_{D_{1}}\right\rangle D_{1} \\
& =\frac{\left(d_{3}\left(d_{2} d_{3}{ }^{\prime}-d_{3} d_{2}{ }^{\prime}\right)+d_{1} d_{3}\left(d_{2}{ }^{2}+d_{3}{ }^{2}\right)\right) T+\left(d_{2}\left(d_{3} d_{2}{ }^{\prime}-d_{2} d_{3}{ }^{\prime}\right)-d_{1} d_{2}\left(d_{2}{ }^{2}+d_{3}{ }^{2}\right)\right) D_{2}}{\left(d_{2}{ }^{2}+d_{3}{ }^{2}\right)^{2}}
\end{aligned}
$$

and by using (2.8) we obtain $\mu_{g}^{D_{1}}$ and complete the proof.

\section{EXAMPLes}

Example 4.1. Let $\alpha$ be the $3^{r d}$ degree polynomial curve in $E^{3}$ given by the following parameterization

$$
\alpha(s)=\left(s, \frac{s^{2}}{2}, \frac{s^{3}}{6}\right) .
$$

From the relations, (2.1), (2.3) and (2.5) the vector elements of Frenet and Flc-frame can be found as

$$
\begin{aligned}
& T(s)=\left(\frac{2}{s^{2}+2}, \frac{2 s}{s^{2}+2}, \frac{s^{2}}{s^{2}+2}\right), \quad N(s)=\left(\frac{-2 s}{s^{2}+2},-\frac{s^{2}-2}{s^{2}+2}, \frac{2 s}{s^{2}+2}\right), \\
& B(s)=\left(\frac{s^{2}}{s^{2}+2},-\frac{-2 s}{s^{2}+2}, \frac{2}{s^{2}+2}\right), \quad D_{2}(s)=\left(\frac{-s^{2}}{\left(s^{2}+2\right) \sqrt{s^{2}+1}}, \frac{-s^{3}}{\left(s^{2}+2\right) \sqrt{s^{2}+1}}, \frac{2 \sqrt{s^{2}+1}}{s^{2}+2}\right) \text {, } \\
& D_{1}(s)=\left(\frac{s}{\sqrt{s^{2}+1}}, \frac{-1}{\sqrt{s^{2}+1}}, 0\right) \text {. }
\end{aligned}
$$


The curvatures and the corresponding unit Darboux vectors $\left(C\right.$ and $\left.C_{W}\right)$ of $\alpha(s)$ according to both Frenet and Flc frame are given in respective order as

$$
\begin{aligned}
\kappa & =\tau=\frac{4}{\left(s^{2}+2\right)^{2}}, \\
d_{1} & =\frac{4 s}{\sqrt{\left(s^{2}+1\right)\left(s^{2}+2\right)^{2}}}, \quad d_{2}=-\frac{4}{\sqrt{\left(s^{2}+1\right)\left(s^{2}+2\right)^{2}}}, \quad d_{3}=\frac{2 s^{2}}{\left(s^{2}+1\right)\left(s^{2}+2\right)^{2}} .
\end{aligned}
$$

The spherical indicatrix of tangent vector $T(s)$ of $\alpha(s)$ is given in Fig. 1

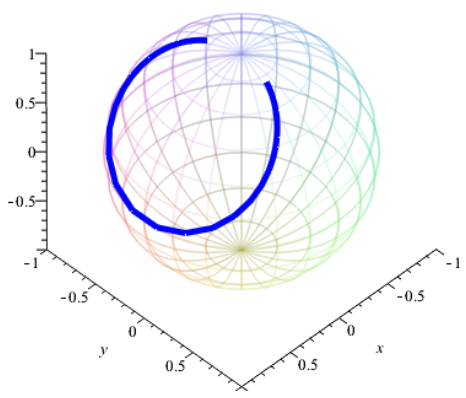

FIGURE 1. Tangent Spherical Image of $\alpha(s)$ (is same for both frames)

The normal and normal like indicatrix curves of $\alpha(s)$ are illustrated in Fig. 2
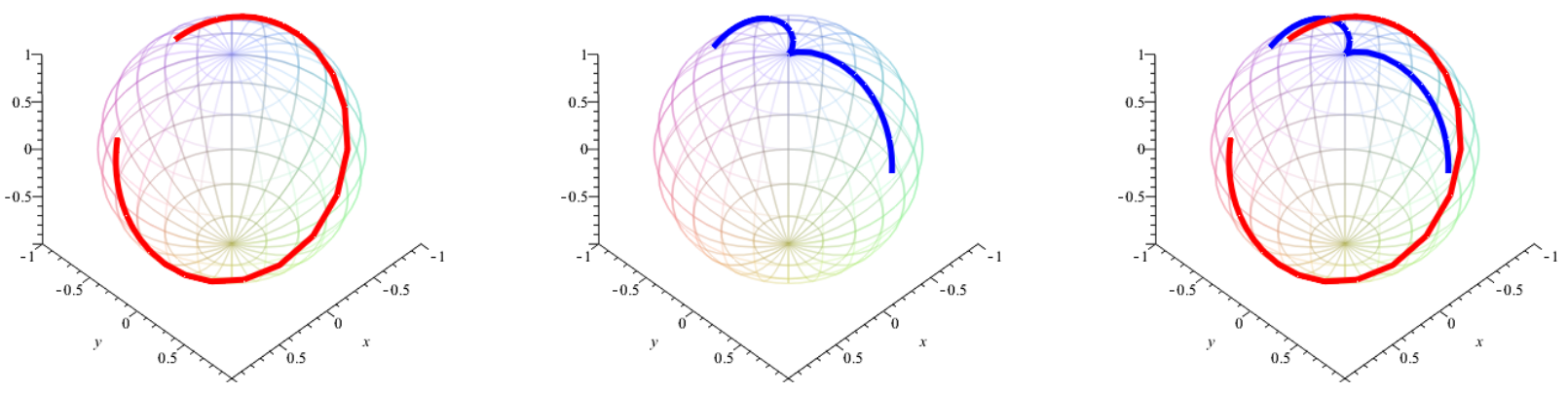

Figure 2. The $N$ - indicatrix (red) and $D_{2}$ - indicatrix (blue) curves of $\alpha(s)$

The binormal and binormal like indicatrix curves of $\alpha(s)$ are illustrated in Fig. 3 

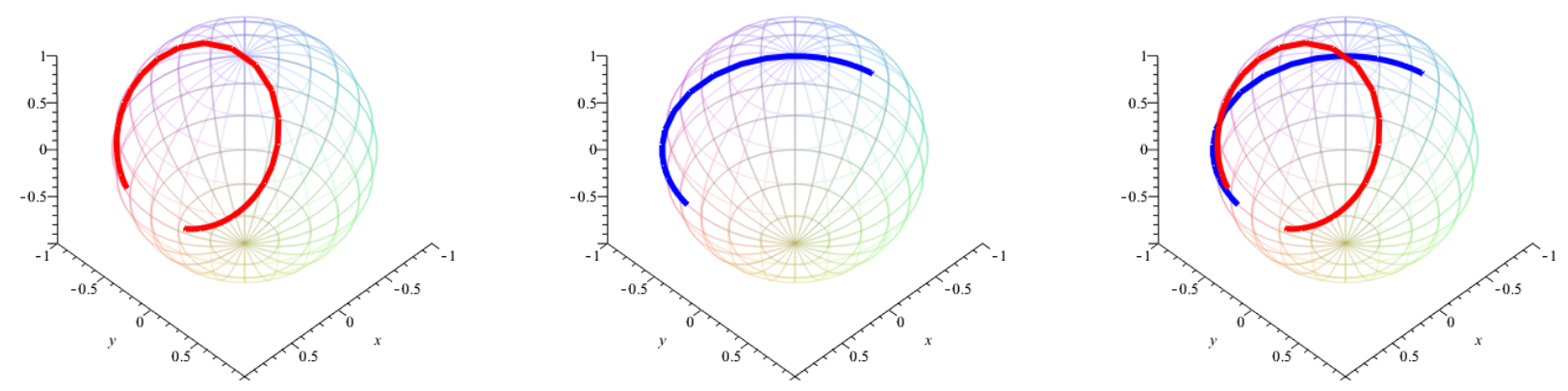

FiguRE 3. The $B$ - indicatrix (red) and $D_{1}-$ indicatrix (blue) curves of $\alpha(s)$

\section{CONFLICTS OF INTEREST}

The authors declare that there are no conflicts of interest regarding the publication of this article.

\section{Authors Contributions Statement}

All authors have contributed sufficiently in the planning, execution, or analysis of this study to be included as authors. All authors have read and agreed to the published version of the manuscript.

\section{REFERENCES}

[1] Bishop, R.L., There is more than one way to Frame a curve, American Mathematical Monthly, 82(3)(1975), $246-251$.

[2] Capın, R., Minkowski Uzayında Küresel Gösterge Eğrileri, Yüksek Lisans Tezi, Gaziantep Üniversitesi Fen Bilimleri Enstitüsü, Gaziantep, 77p, 2016.

[3] Dede, M., A new representation of tubular surfaces, Houston J. Math. 45(3)(2019), 707-720.

[4] Farouki, R.T., Han, C.Y., Manni, C., Sestini, A., Characterization and construction of helical polynomial space curves, Journal of Computational and Applied Mathematics 162(2004), 365--392.

[5] Gökyeşil, D., Dual Uzayda Bazı Eğrilerin Dual Bishop Çatısına Göre Karakterizasyonları, Yüksek Lisans Tezi, Manisa Celal Bayar Üniversitesi Fen Bilimleri Enstitüsü , Manisa, 65p, 2018.

[6] Hacısalihoğlu, H.H., Diferansiyel Geometri, Ankara Üniversitesi Fen Fakültesi, Ankara, 1993.

[7] Kula, L., Yayli, Y. On slant helix and its spherical indicatrix, Appl. Math. Comput., 169(1)(2005), 600-607.

[8] Larson, R., Elemantary Linear Algebra, The Pennsylvania State University, Boston, 2012.

[9] O’Neill, B., Elementary Diferential Geometry. New York, Academic Press Inc., 1966.

[10] Şenyurt, S., Natural lifts and the geodesic sprays for the spherical indicatrices of the Mannheim partner curves in $E^{3}$, International Journal of the Physical Sciences, 7(16)(2012), 2414-2421.

[11] Şenyurt, S., Özgüner, Z., Bertrand eğri çiftinin küresel göstergelerinin geodezik eğrilikleri ve tabii liftleri, Ordu Üniv. Bil. Tek. Derg., 3(2)(2013), 58-81.

[12] Yılmaz, S., Özyılmaz, E., Turgut, M., New spherical indicatrices and their characterizations, An. Şt. Univ. Ovidius Constanta., 18(2)(2010), 337-354. 